\title{
Penile skin preservation technique for reconstruction surgery of penile paraffinoma
}

\author{
Jong Sung Kim ${ }^{1}$, Yu Seob Shin ${ }^{2}$, Jong Kwan Park ${ }^{2}$ \\ ${ }^{1}$ Department of Urology, Kim Jong Sung Urological Clinic, Iksan, ${ }^{2}$ Department of Urology, Chonbuk National University Medical School, Research Institute of Clinical \\ Medicine of Chonbuk National University-Biomedical Research Institute and Clinical Trial Center of Medical Device of Chonbuk National University Hospital, Jeonju, \\ Korea
}

Purpose: Penile resurfacing with various scrotal flaps is a simple, effective, and reliable reconstructive armamentarium to treat penile paraffinoma. However, scrotal flaps often result in heavy hair-bearing penile skin causing dyspareunia or shortening of penile length. We introduce a new penile skin preservation technique for reconstruction surgery of penile paraffinoma.

Materials and Methods: From March 2007 to April 2018, 23 patients underwent excision of paraffinoma with the penile skin preservation technique. Complete removal of the subcutaneous tissue was done, including paraffinoma. No. 10 surgical blades were used to peel off the skin as thin as possible in order to get paraffinoma-free skin. The preserved penile skin was given an end to end anastomosis to the circumferential incision site. Multiple anchoring fixation sutures of the penile skin were made on the underlying tissue. Dressing of chopped gauze soaked with antibiotic saline solution was done on the skin to help preserve it and keep it fixed on the penis.

Results: The skin survived completely without necrosis in 19 patients (82.6\%), with the mean duration of healing being 21.9 days. The penis after the operation looked natural in color and shape. Partial necrosis after the operation was shown on 4 patients (17.4\%), whom were all later treated with the scrotal skin free graft. The final results were successful without any cosmetic or functional complications, such as skin contracture.

Conclusions: Penile resurfacing with the penile skin preservation technique is a new effective reliable method for skin-intact paraffinoma which produces good functional and cosmetic results.

Keywords: Foreign bodies; Penis; Reconstructive surgical procedures; Skin

This is an Open Access article distributed under the terms of the Creative Commons Attribution Non-Commercial License (http://creativecommons.org/licenses/by-nc/4.0) which permits unrestricted non-commercial use, distribution, and reproduction in any medium, provided the original work is properly cited.

\section{INTRODUCTION}

In Asian and Eastern European cultures, a big penis has been frequently considered as a symbol of power. Injection of foreign materials under the penile skin in order to make the penis bigger has been reported in several countries of Eastern Asia [1-3]. However, the injections are usually followed by complications, including penile deformity, skin necrosis, decreased erectile function, and painful intercourse $[4,5]$. The mass induced by granulomatous skin and subcutaneous reactions is called vaselinoma or paraffinoma. The definitive treatment is the complete removal of the involved skin and subcutaneous tissue, and resurfacing the penile shaft. Penile resurfacing with various scrotal

Received: 5 November, 2018 - Accepted: 30 December , 2018

Corresponding Author: Yu Seob Shin

Department of Urology, Chonbuk National University Medical School, 20 Geonji-ro, Deokjin-gu, Jeonju 54907, Korea

TEL: +82-63-250-1565, FAX: +82-63-250-1564, E-mail: ball1210@hanmail.net

ORCID: https://orcid.org/0000-0002-1126-3821 
flap techniques is a relatively simple, effective, and reliable single-stage reconstructive armamentarium for paraffinoma [6]. However, scrotal flaps often result in heavy hair-bearing reconstructed penile skin causing dyspareunia and in the shortening of penile length in its flaccid state due to both the inherent contractile property of the scrotum and the scrotal flap being lengthened beyond its natural state to cover the penis. We introduce a new repairing technique, a penile skin preservation technique for reconstruction surgery of penile paraffinoma, which is expected to be effective cosmetically and functionally without complications, such as a hairy penis or a short penis.

\section{MATERIALS AND METHODS}

\section{Patients}

From March 2007 to April 2018, 23 patients underwent penile skin preservation technique during the removal of penile paraffinoma. We obtained approval for the study from the Institutional Review Board at Chonbuk National University Hospital. Written informed consent was obtained from each patient. Also, this study was conducted in accordance with the Good Clinical Practice and the International Conference on Harmonization guidelines, and in conformity with the ethical principles of the Declaration of Helsinki. Most patients had a severe penile deformity from just under the coronal sulcus to the penoscrotal junction due to foreign body granulomas and inflammation (Fig. 1A). Foreign body granulomas had partially invaded the scrotum and the prepubic fat layer in patients (Table 1). The indication of penile skin preservation technique is movable skin from the underlying tissue, namely no fixation of the penile skin to the underlying tissue for at least one quarter of the skin indicating no severe infection on that part of the area. The average age and mean follow-up periods are listed in Table 1.

\section{Operative technique}

All operations were performed by one surgeon who is experienced to urological surgery. Under regional anesthesia, a Foley catheter is inserted into the bladder. A circumferential incision was made at the skin just proximal to the corona of the penis. No. 10 surgical blades were used to peel off the skin as thin as possible in order to get paraffinoma free skin (Fig. 1B). Complete removal of the subcutaneous tissue, including paraffinoma, was performed as usual (Fig. 1C). We minimized the use of electrocautery to avoid damage to the devascularized penile skin. Meticulous coagulation of the penile shaft was done. Preserved penile skin has been given an end to end anastomosis to the circumferential incision site. Numerous fenestrations of 3 to $5 \mathrm{~mm}$ in length on the skin were made for drainage of tissue fluid or blood. As the most important procedure in this operation, multiple anchoring fixation sutures of 4-0 nylon between the penile skin and the equivalent underlying penile bed was done (Fig. 1D). Dressing of chopped gauze soaked with antibiotic saline solution was made on the skin for fixation. The chopped gauze dressing was fixated with multiple 4-0 nylon sutures between the distal and proximal end of the devascularized penile skin. Vaseline gauze was wrapped on the gauze dressing. Another wet gauze dressing was done on the vaseline gauze wrapping, which ended with

Table 1. Patients characteristics

\begin{tabular}{lc}
\hline \multicolumn{1}{c}{ Variable } & Value \\
\hline Patients & 23 \\
Age $(y)$ & $45.3 \pm 8.6$ \\
Mean duration of wound healing (d) & $21.9 \pm 12.2$ \\
Location of paraffinoma & 21 \\
Penis & 2 \\
Penis and scrotum & $12.2 \pm 7.9$ \\
Mean follow-up period (mo) & \\
Major complication & 4 \\
Partial graft necrosis needed 2nd free graft
\end{tabular}

Values are presented as number only or mean \pm standard deviation.
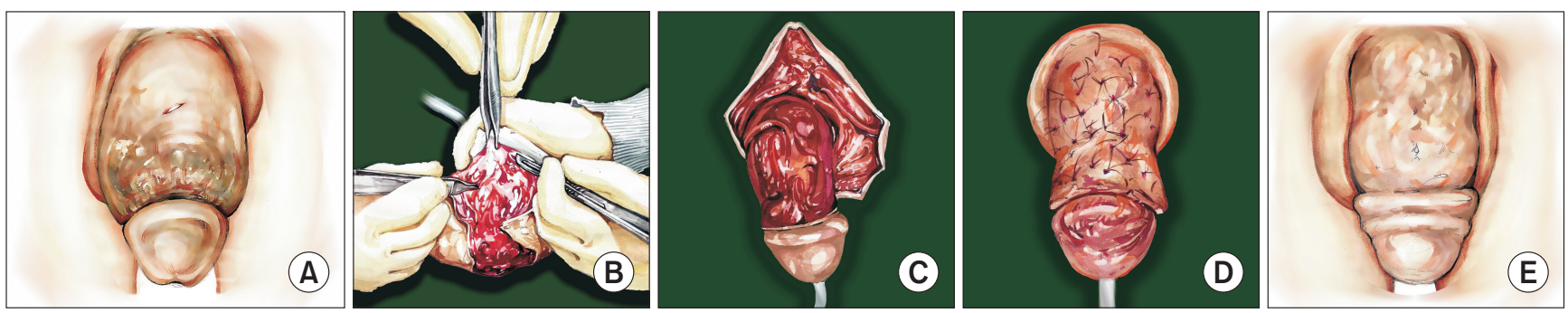

Fig. 1. Schematic view of operative stages. (A) Penile deformity due to penile paraffinoma. (B) Peel off the skin as thin as possible in order to get paraffinoma free skin. (C) Complete removal of paraffin. (D) Multiple anchoring fixation sutures between the penile skin and penile bed was done. (E) The penile skins survived completely without skin contracture. 
a Coban compression dressing. Patients were discharged later on the day of operation. Five days after operation, the dressing was carefully undressed after wetting the gauze in order to avoid the skin from being detached from the underlying bed. Vaseline gauze dressing applied daily until the complete survival of the skin. Penile skin necrosis which could not be healed by dressing was treated with scrotal skin free graft.

\section{RESULTS}

The penile skins survived completely without skin contracture in 19 patients (82.6\%) 3 weeks after surgery (Table 1, Fig. 1E). Partial necrosis which needed wound dressing up to 2 months at the clinic happened in the remaining 4 patients (17.4\%) (Table 1). The necrosis always developed around the glans. After healing from the necrotic skin, fibrotic scar tissue resulted in the penile curvature near the coronary sulcus in 2 patients. The complications were successfully treated with scrotal skin free graft 2 months after the first operation in all 4 cases. No curvature or graft contracture was developed in any patient after the second procedure.

In the case of the 19 patients who successfully underwent the penile skin preservation technique without necrosis, the temporarily decreased penile sense were recovered in the following 6 months after the operation for all of them (Fig. 2). As time passed, the implanted skin became more and more tensile without contracture in all 19 successful cases, and satisfactory sexual activity without feeling tightness were possible for all patients. No patient among those complained of either penile pain during coitus or cosmetic problems during follow-up.
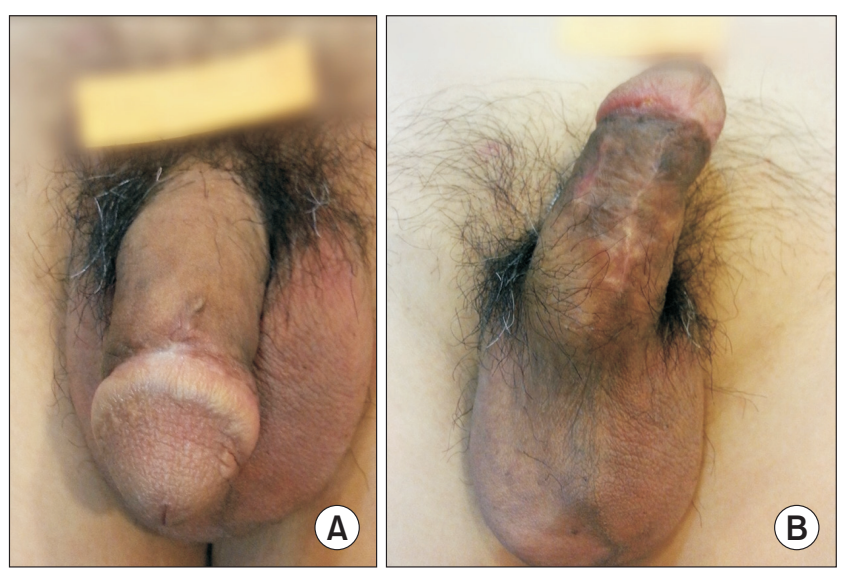

Fig. 2. A 2 months after penile resurfacing with the penile skin preservation technique. (A) Dorsal side. (B) Ventral side.

\section{DISCUSSION}

The penile paraffinoma or vaselinoma can be variously confined to distal shaft, mid-shaft, or whole penile shaft with wide migration of paraffin or vaseline to infrapubic area, scrotum, and/or glans. The paraffinoma can be confined to a partial or full layer of Dartos fascia, or can infiltrate the skin, and deep all the way to Buck's fascia. Conventional penile resurfacing procedures differ depending on the width and depth of the paraffin infiltration. Mild paraffin infiltration in depth and width is easily treated with excision of paraffinoma and simple closure [7]. In cases of deeper infiltrations with severe paraffinoma, various scrotal flap procedures are used as reconstructive armamentariums since the scrotum is easily available, extensile, and has the same color as the penile skin [8]. However, in cases of small or hairy scrotums, scrotal flap is not appropriate for penile resurfacing after paraffinoma excision. Hairy scrotal flap cause dyspareunia and scrotal flap usually cause the shortening of penile length in flaccid state due to both the inherent contractile property of the scrotum and the scrotal flap being lengthened beyond its natural state to cover the penis. Considering the patients' primary intension of injecting paraffin were the elongation of penile length and a more satisfactory sexual intercourse, a hairy or shorter penis due to the operation is a definite disadvantage. Previously, we reported the introduction of a new repair technique for penile paraffinoma without the use of T-style anastomosis at the ventral skin of the penis [9]. We experienced excellent results using this technique. However, heavy hair in the scrotal skin can be a cosmetic and functional problem after scrotal flap surgery. For patients with abundant hair problems, they later have to remove the hair follicles to achieve comfort. However, new penile skin preservation technique for penile paraffinoma has several cosmetic advantages compared to previous surgical methods. It keeps the penis form intact without skin contracture or heavy hair. Devascularized penile skin is very extensible in nature with no hair-bearing, so we theorized that preserving the penile skin would overcome the demerits of the conventional scrotal flap, such as dyspareunia, or a short flaccid penis.

Full-thickness or split-thickness skin graft from the inguinal area or abdomen after paraffinoma excision is feasible [10]. But those grafts are not as extensible as penile skin, and also are different in color from penile skin. Moreover, these sorts of grafts commonly cause skin graft contracture mostly due to the fact that the skin not originating from that area. Original penile skin naturally doesn't have that type of disadvantage, thus having a very 
low possibility of penile skin graft contracture as seen in our end result. In our 23 cases of penile skin preservation technique, complete skin survival was achieved in 19 cases $(82.6 \%)$. The time for recovery was usually within 3 weeks. No skin contracture developed. We believe that those results can recommend penile resurfacing with penile skin preservation technique as a good armamentarium for severe paraffinoma that infiltrated no more than approximately three quarters of the penile skin.

In our 23 cases of penile skin preservations, partial necrosis which needed a skin graft developed in 4 cases (17.4\%). We believe that the main cause of necrosis is the infiltration of paraffin in the remaining skin. This is usually due to the incomplete excision of paraffinoma from the skin which can be overcome by meticulous excision of paraffin-infiltrated tissue. No. 10 surgical blades are recommended for peeling off the skin for the same reason. Necrosis usually develops at the glandular side. Glandular infiltration of paraffinoma needs partial glandular excision. In that case, we believe that the excised glandular margin in the penis can produce dead space under the anastomotic suture, leading to necrosis. Meticulous anchoring sutures to the underlying bed at the dead space is essential to avoid graft necrosis in that area. Wet graft dressing is done for 4 weeks in some cases before the necrotic tissue debridement for a skin free graft.

Severe paraffinoma that infiltrated most of the skin is a strong contraindication for this technique. If peeled devascularized skin is infiltrated by paraffin, such an area should be excised before covering the skin on the shaft. Easily movable skin on the paraffinoma usually predicts very little to no infiltration of paraffin into the skin, which is a good indication for this penile resurfacing operation. Peeling the skin to get paraffin-less skin takes time and endurance. If the condition of the devascularized skin is poor or peeling the skin is not easy due to heavy paraffin infiltration on the skin, the surgical plan can be switched from the penile skin preservation technique to the conventional scrotal flap at any time during operation under regional anesthesia. It should be noted that this method of confirming which skin to preserve and which skin to remove is based on pure observation in the surgery room rather than a more scientific approach.

Limitations of our study include: 1) the retrospective study design could have affected the primary acquisition of data; 2) our study was restricted to only those men who had been diagnosed with skin-intact penile paraffinoma. A greater number of studies that include a larger number of patients and multi-institution studies are warranted in future. Despite these limitations, to the best of our knowledge, the present study is the first reported, new penile skin preservation technique for reconstruction surgery of penile paraffinoma. Additionally, many uninformed patients are candidates to accept the paraffin injection procedure, we urge that urologist try to give public information for healthy sexual life is important [11-16].

\section{CONCLUSIONS}

Penile resurfacing with penile skin preservation technique is a new effective and reliable method for skinintact penile paraffinoma, which produces a functional and cosmetically sound result. Judicious choice of candidates, acquiring paraffin-free penile skin, sewing meticulous anchoring sutures (especially around glans), and maintaining a firm fixation until full recovery are crucial for the success of penile skin preservation technique for reconstruction surgery of penile paraffinoma.

\section{CONFLICTS OF INTEREST}

The authors have nothing to disclose.

\section{ACKNOWLEDGMENTS}

The authors thank In A Yoon, with thanks, for schematic figures of the operative findings. This paper was partially supported by a Fund from the Biomedical Research Institute, Chonbuk National University Hospital.

\section{REFERENCES}

1. Manny T, Pettus J, Hemal A, Marks M, Mirzazadeh M. Penile sclerosing lipogranulomas and disfigurement from use of "1Super Extenze" among Laotian immigrants. J Sex Med 2011;8:3505-10.

2. Rosenberg E, Romanowsky I, Asali M, Kaneti J. Three cases of penile paraffinoma: a conservative approach. Urology 2007;70:372.e9-10.

3. Nyirády P, Kelemen Z, Kiss A, Bánfi G, Borka K, Romics I. Treatment and outcome of vaseline-induced sclerosing lipogranuloma of the penis. Urology 2008;71:1132-7.

4. Cohen JL, Keoleian CM, Krull EA. Penile paraffinoma: selfinjection with mineral oil. J Am Acad Dermatol 2002;47(5 Suppl):S251-3.

5. Chon W, Koo JY, Park MJ, Choi KU, Park HJ, Park NC. Paraffin granuloma associated with buried glans penis-induced sexual and voiding dysfunction. World J Mens Health 
2017;35:129-32.

6. Jeong JH, Shin HJ, Woo SH, Seul JH. A new repair technique for penile paraffinoma: bilateral scrotal flaps. Ann Plast Surg 1996;37:386-93.

7. Dellis AE, Nastos K, Mastorakos D, Dellaportas D, Papatsoris A, Arkoumanis PT. Minimal surgical management of penile paraffinoma after subcutaneous penile paraffin injection. Arab J Urol 2017;15:387-90.

8. Zhao YQ, Zhang J, Yu MS, Long DC. Functional restoration of penis with partial defect by scrotal skin flap. J Urol 2009;182:2358-61.

9. Shin YS, Zhao C, Park JK. New reconstructive surgery for penile paraffinoma to prevent necrosis of ventral skin. Urology 2013;81:437-41.

10. Kim SW, Yoon BI, Ha US, Kim SW, Cho YH, Sohn DW. Treatment of paraffin-induced lipogranuloma of the penis by bipedicled scrotal flap with Y-V incision. Ann Plast Surg 2014;73:692-5.

11. Yang DY, Ko K, Lee SH, Cho JS, Lee SK, Shin TY, et al. Postmicturition dribble is associated with erectile dysfunction in middle-aged and older men with lower urinary tract symp- toms. World J Mens Health 2018;36:263-70.

12. Park HJ, Lee KS, Lee EK, Park NC. Efficacy and safety of a mixed extract of trigonella foenum-graecum seed and lespedeza cuneata in the treatment of testosterone deficiency syndrome: a randomized, double-blind, placebo-controlled clinical trial. World J Mens Health 2018;36:230-8.

13. Yeo JK, Cho SI, Park SG, Jo S, Ha JK, Lee JW, et al. Which exercise is better for increasing serum testosterone levels in patients with erectile dysfunction? World J Mens Health 2018;36:14752.

14. Moon DG. Changing men's health: leading the future. World J Mens Health 2018;36:1-3.

15. Won JE, Chu JY, Choi HC, Chen Y, Park HJ, Dueñas HJ. Safety and effectiveness of once-daily tadalafil $(5 \mathrm{mg})$ therapy in Korean men with benign prostatic hyperplasia/lower urinary tract symptoms in a real-world clinical setting: results from a post-marketing surveillance study. World J Mens Health 2018;36:161-70.

16. Park HJ. The role of the urologist in men's health. World J Mens Health 2017;35:57-8. 